\title{
Vigueur de croissance de deux variétés de légumes installées sur sols contaminés à Lubumbashi (RD Congo).
}

\author{
Mununga Katebe Félicien ${ }^{a \infty b^{*}}$, Mwilambwe Kapoma Xaviera, N'tambwe Nghonda Dieu-donnéb, Maloba \\ Kazembe Jean-Pierrec, Banza llunga Meschackc, Chola Kazembe Augustind, Lwanga Kapwaye Michaeld, \\ Mpundu Mubemba Mulambi Michela,b. \\ aDépartement de phytotechnie, Faculté des Sciences Agronomiques, Université de Lubumbashi, BP : 1825 Lubumbashi, \\ RD Congo ; \\ 'Département de Gestion des Ressources Naturelles Renouvelables. Faculté des Sciences Agronomiques, Université de \\ Lubumbashi, BP : 1825 Lubumbashi, RD Congo ; \\ ${ }^{\circ}$ Centre de Recherche Agro-alimentaire(CRAA) Lubumbashi, RD Congo. \\ dDépartement d'économie, Faculté des sciences économiques, Université de Lubumbashi, BP : 1825 Lubumbashi, RD \\ Congo. \\ *Auteur correspondant : munungakatebe@gmail.com , +243815778141.
}

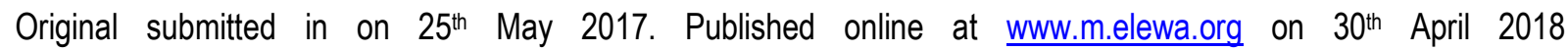
https://dx.doi.org/10.4314/jab.v124i1.1

\section{RESUME}

Objectifs : L'objectif de cette étude était d'évaluer le comportement de deux variétés de deux légumes installés sur les sols contaminés en ETMs dans la région de Lubumbashi.

Méthodologie et résultats: Deux essais suivant un dispositif factoriel $4 * 2$ ont été installés, dont le facteur principal est constitué de deux variantes de laitue et de Gombo (variété améliorée et celle locale) et le facteur secondaire qui 4 variantes de sol dont celui de (de Kipushi, de Pengapenga, de Luwowoshi (Ruashi) ainsi que celui de la ferme Kassapa). Les résultats obtenus dans cette étude montrent que les sols sont contaminés en cuivre et la laitue ayant évoluée sur ces substrats présentent des teneurs en cuivre très élevées dépassant la norme, la variété locale avait présenté un bon comportement contrairement à la variété améliorée venant de la France sur ce qui est de la survie, du taux de levée.

Conclusion et applications des résultats: la variété locale de laitue et de Gombo sont les mieux appropriées pour les sols contaminés, toutes fois l'application des amendements organiques et calcaires permettrait d'avantage de réduire la mobilité de cuivre dans le sol et le transfert de ce dernier vers les parties aériennes. Mots clés : Variété, contamination, gombo, laitue.

\begin{abstract}
Objectives: The objective of this study was to evaluate the behavior of two varieties of two vegetables installed on soils contaminated with ETMs in the Lubumbashi region.

Methodology and results: Two tests using a factorial $4{ }^{*} 2$ device were installed, the main factor of which consists of two variants of lettuce and okra (improved variety and local variety) and the secondary factor which 4 soil variants including that of (Kipushi, Pengapenga, Luwowoshi (Ruashi) and Kassapa Farm). The results obtained in this study show that the soils are contaminated with copper and lettuce having evolved on these
\end{abstract}


substrates have very high copper contents exceeding the norm, the local variety had exhibited a good behavior contrary to the improved variety coming from France. on what is survival, the rate of emergence.

Conclusion and applications des results: the local variety of lettuce and okra are the most appropriate for contaminated soils, all the time the application of organic and limestone amendments would better reduce the mobility of copper in the soil and the transfer of the latter towards the aerial parts.

Keywords: Variety, contamination, okra, lettuce.

\section{INTRODUCTION}

Vers les années 2030, la population mondiale va augmenter de 3 milliards d'individus, et cette augmentation sera beaucoup plus remarquable dans les pays en développement à plus de $95 \%$ (Mougeot et Moustier, 2004). Mais cette montée démographique concerna surtout les zones urbaines. Les études effectuées par (Padilla, 2004), ont montrées qu'il existe une corrélation entre l'urbanisation et le niveau de pauvreté de la population. Par ailleurs, cette augmentation va entrainer des augmentations en besoins alimentaires, c'est ainsi que d'ici 2030 , la production devra aussi doubler pour satisfaire aux besoins de la population. (Mougeot et Moustier, 2004). Le développement de l'agriculture urbaine s'observe dans des nombreuses villes d'Afrique subsaharienne avec prédominance du système maraichère urbain et périurbain, malheureusement la ville de Lubumbashi, regorge diverses usines hydrométallurgiques et fonderies du cuivre et du cobalt ; mais ces dernières rejettent les déchets et les effluents ainsi que des sous-produits enrichis en éléments traces métalliques ou en métalloïdes notamment (Cadmium, Plomb, Zinc, Arsenic), ou en d'autres composés soufrés (Leupin \& Hug, 2005). Alors que ces rejets ont un effet néfaste sur l'environnement notamment : l'air, l'eau, le sol accumulent des contaminants, c'est ainsi qu'on observe un disfonctionnement de leurs propriétés et causant à leur tours des troubles aux êtres vivants ainsi qu'à leurs environnements (Bruneau, 1983 ; Tembo et al., 2005 ; Leveque, 2014). Il a été recensé une dizaine de type de perturbation engendrés par l'exploitation minière dans l'hinterland minier du Katanga, avec l'accumulation des polluants dans les sols agricoles (Le Teinturier et al, 1999 ; Baker et Malaise, 1999). Les activités minières dans la ville de Lubumbashi, en dépit du fait qu'elles permettent de résorber le chômage en donnant de l'emploi à la population locale, provoquent la pollution des eaux et du sol, détériorent la qualité des denrées alimentaires par l'émission des produits toxiques (Peter, 1999). En effet, l'exploitation minière, le traitement des minerais, leur stockage, la manufacture des produits finis et la mise en décharge des déchets de ces activités entrainent l'émission et la dispersion des particules métalliques dans l'environnement. Le plus souvent, elles s'accumulent à proximité même du lieu d'émission ; leur chronicité amène les sols à des stades de déséquilibre biologiques plus ou moins avancés agissant par la toxicité sur la microflore des couches superficielles du sol (Impes et al., 1999). L'activité industrielle liée à l'exploitation des minerais riches en métaux, avait provoqué la pollution des sols en éléments traces métalliques (Baize et Tercet, 2002). La contamination des sols présente un risque de toxicité pour les êtres vivants et l'homme à travers la chaine alimentaire. Elle entrave la croissance et la viabilité de la végétation, par conséquent, la mise en place des cultures y est ardue voir impossible, selon le degré de pollution mais aussi risqué par l'accumulation des contaminants par les végétaux et son transfert dans la chaine alimentaire (Mach et Baize, 2004, Bourrelier et Bertelin, 1998 ; Mpundu, 2010). Cette étude a été initiée en vue d'évaluer le comportement de deux variétés de deux légumes (variété locale et améliorée venant de la France) de la laitue et du gombo installées sur les sols contaminés en cuivre dans la région minière du Haut-Katanga. Ce présent essai avait comme objectifs spécifiques d'évaluer les comportements de deux légumes de nos deux variétés de laitue et du gombo installées sur les sols contaminés en ETMs d'une part et de déterminer la variété la plus résistante aux sols contaminés et donc qui a un rendement meilleur d'autre part. Déterminer la teneur de cuivre contenus dans différents sols dans 
la région de Lubumbashi; Comparer le comportement de deux variétés de gombo installées sur sol contaminés en cuivre. Deux hypothèses ont été émises dans cet essai (i) les fortes concentrations en Etms dans le sol entraineraient un ralentissement de croissance des plantes, parfois leurs disparition ; (ii) les variétés locales seraient les

\section{MATÉRIELS ET MÉTHODES}

Matériels biologiques : cette étude a portée sur deux variétés de deux légumes notamment les plantes d'Abelmoschus esculentus (Gombo) et de Lactuca sativa (Laitue) ont été utilisées pour notre expérimentation. Les variétés améliorées génétiquement provenant de la France pour la laitue ainsi que pour le Gombo ont été achetées au marché Kenya et les variétés locales cultivées couramment dans la région de Lubumbashi ont été achetées au marché Mzee.

Méthodes : ces essaies ont été conduits au champ expérimental de la faculté des sciences agronomiques de l'université de Lubumbashi. Ce site expérimental est situé à $1230 \mathrm{~m}$ d'altitude dans le carré formé par les latitudes $11^{\circ} 39^{\prime}$ et $11^{\circ} 42^{\prime}$ sud et les longitudes $27^{\circ} 27^{\prime}$ et $27^{\circ} 28^{\prime}$ de longitude Est. Deux essaies à deux facteurs ont été installés, le facteur principal est constitué de deux variantes de laitue et d'aubergine (variété améliorée et celle locale) et le facteur secondaire qui avait 4 variantes de sol dont celui (de Kipushi, de Pengapenga, de Luwowoshi(Ruashi) ainsi que celui de la ferme Kassapa). Notre essai a été conduit dans les conditions de culture comparable presque à celles paysannes dans le jardin botanique de la faculté des sciences agronomiques suivant un dispositif en bloc complet randomisé comprenant 4 répétitions.

Analyse chimique et expérimentation : un échantillon composite a été collecté pour chaque type de sol à une profondeur de $0-20 \mathrm{~cm}$. Ces échantillons de sols tout comme ceux des plantes récoltées ont été acheminés au laboratoire Agro-pédologique de la faculté des sciences agronomiques de l'université de Lubumbashi pour l'analyse en vue de déterminer les quantités de cuivre dans ces différents sols. Les échantillons des plantes récoltées ont été lavés à l'eau de robinet, puis placés dans les enveloppes. Puis les enveloppes ont été mis dans l'étuve à une température de $40^{\circ} \mathrm{C}$ pendant 24 heures pour un séchage des échantillons, et enfin ces derniers ont été broyés et mises dans des petites boites. Après la collecte des échantillons de sols pour les différents traitements, les opérations préparatoires aux analyses de laboratoire ont été réalisées notamment plus adaptées aux fortes concentrations en Etms que la variété améliorée venant de la France ; (iii) la teneur en cuivre serait plus élevée dans le sol de Ruashi, par rapport aux autres sols types de sols. La variété locale s'adapterait mieux aux stress nutritionnel par rapport à la variété améliorée.

l'émiettement de grosses particules après séchage, le criblage et le pesage. Toutes ces opérations finies, les échantillons des sols ont été mis dans des petites boites portant les indications se rapportant aux sites où les sols ont été prélevés. Les ETMs dans les végétaux comme dans les sols ont été déterminés à l'aide d'un appareil à fluorescence $X$ de marque OLYMPUS. Cette analyse chimique a consisté à doser les différents éléments chimiques. Le dosage a été réalisé par spectrométrie de fluorescence $X$. En effet, la fluorescence $X$ est une méthode analytique non-destructive des échantillons dont le principe est basé sur l'irradiation d'un échantillon par des rayons $X$. Lorsque l'échantillon absorbe les rayons incidents, celui-ci émet une fluorescence ou des radiations situées dans le domaine des rayons $X$. Les observations ont porté sur les paramètres végétatifs suivants :

Taux de reprise(\%) : Déterminé par le rapport entre le nombre de plante reprise et le nombre de plante repiquée multiplié par 100 ;

Taux de levée(\%): Déterminé par le rapport entre le nombre de plante germée et le nombre de plantes semées multiplié par 100 ;

Le taux de survie (\%) : Déterminé par le rapport entre le nombre de plante survivant après la reprise et le nombre de plante reprise multiplié par 100 ;

La taille des plantes $15 ; 30$ et 45 jours après repiquage $(\mathrm{cm})$ : Déterminée par la mesure graduée à l'aide de la latte :

Le nombre de feuilles $15 ; 30 ; 45$ jours après repiquage : Déterminé par le comptage des feuilles par plante ;

La teneur en Cu et la phytodisponibilité : Déterminée par la mesure au laboratoire de la quantité de cuivre bioaccumulée par les plantes.

Traitement des données : Le logiciel $R$ a été utilisé pour les traitements des données. L'analyse de variance (ANOVA) et la séparation des moyennes (test de Tukey) ont été utilisées pour déterminer les différences entre les traitements à l'aide du logiciel Minitab version 16. Les résultats d'analyse des échantillons des sols et des végétaux, ont été confronté aux normes utilisées dans la 
plus part des pays dans le monde. Pour les échantillons des sols, le seuil des valeurs en ETM extraits à l'acide éthylène diamine-tétra-acétique (EDTA) pour tous les
ETM étudiés. De ce fait les résultats obtenus sont comparés aux seuils selon les normes internationales, de même pour les résultats des végétaux.

\section{RESULTATS}

Concentrations en ETMs dans les racines et les feuilles de la laitue d'Abelmoschus esculentus et de Lactuca sativa.

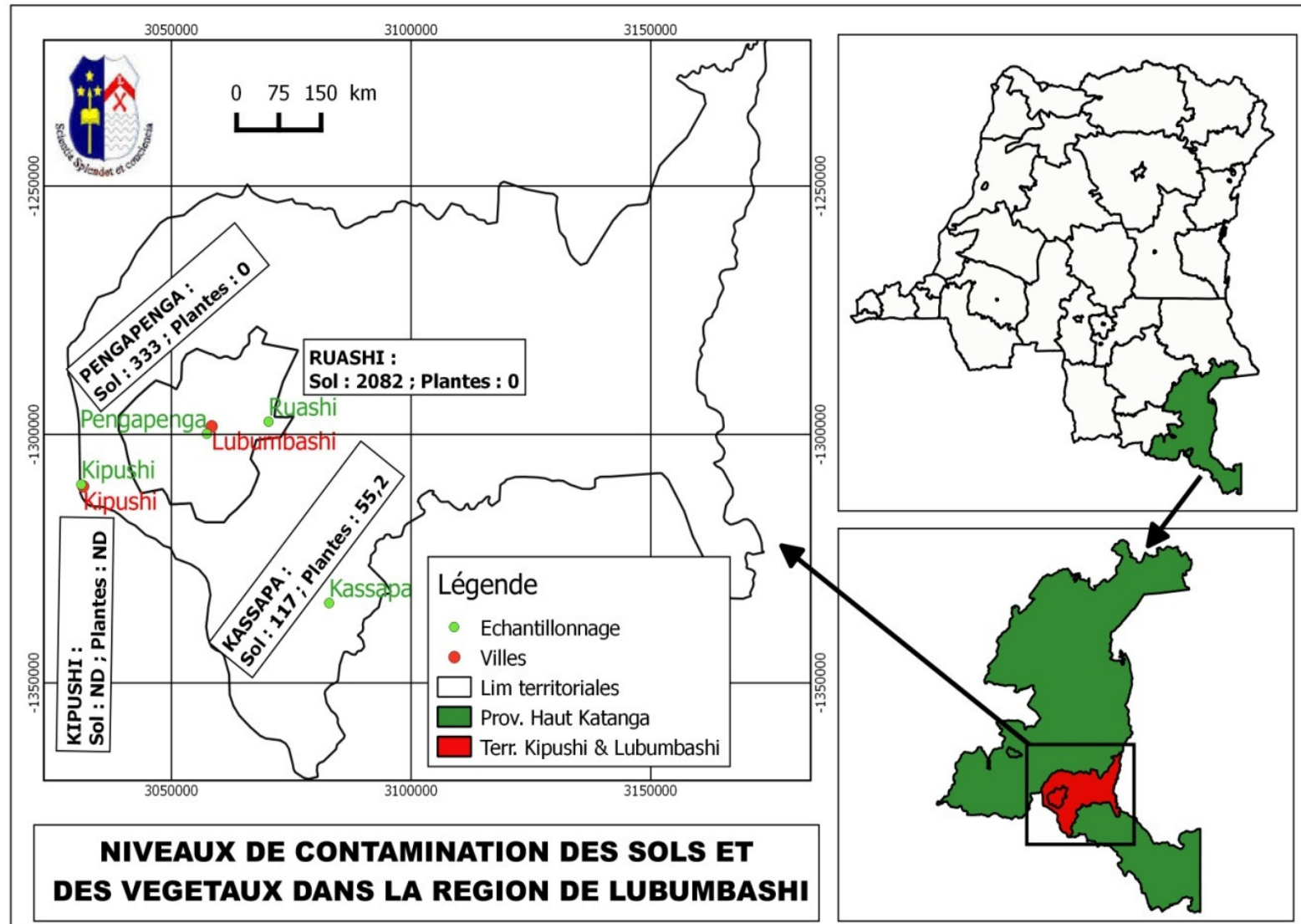

Figure 1 : Carte de niveau de contamination des sols et des végétaux dans la région de Lubumbashi.

Légende : N.D : Non déterminé ; ppm : parti par million.

Partant de la figure 1, les résultats de laboratoire montrent que de tous les quatre types de sol utilisés, le sol de Ruashi avait présenté de teneurs 20 fois supérieures à la norme, alors que le sol de Pengapenga avait présenté de teneurs 3 fois supérieures à la norme, mais le sol de Kassapa considéré comme témoin avait excéder légèrement la norme; contrairement au sol de Kipushi qui n'avait pas présenté de traces de contamination cuprique. Les résultats d'analyse de laboratoire, montrent que les concentrations du cuivre que renferme la laitue dépassent près de cinq fois la teneur normale (seuil de toxicité) pour le sol de Kassapa, alors que le sol de Kipushi n'a pas présenter de traces de contamination cuprique, contrairement au sol de Ruashi et Pengapenga où aucune survie n'a été obtenue à la fin de l'expérimentation.

Taux de reprise et taux de survie de la laitue: Les résultats de l'analyse de la variance révèlent des différences significatives entre les traitements $(p=0,000)$ pour ce qui est de la levée à 7 jours ainsi que de la survie à toutes les dates d'observations. Par rapport à l'utilisation des types de sols, le meilleur taux de levée (100\%) a été obtenu avec le sol de Kipushi, les sols de Kassapa et Ruashi ont présentés des taux de levée intermédiaires, alors que le taux de levée le plus faible a été obtenue avec les sols de Pengapenga. L'analyse de 
la variance montre que l'effet variété n'a pas influencé significativement la levée à toutes les dates d'observation et cela pour toutes les deux variétés. Quant à la variété le meilleur taux de levée a été obtenu avec la variété locale. Cependant l'interaction entre traitement et variété a influencé significativement la levée à 6 jours $(p=0.009)$. Par rapport à l'utilisation des types de sols, le meilleur taux de survie a été obtenu avec le sol de Kipushi, les sols de Kassapa et Rashi ont présenté des taux de survie intermédiaires, alors que le taux de survie le plus faible a été obtenue avec le sol de Pengapenga. L'effet variété n'a pas influencé significativement la survie de la laitue à toutes les dates des semis. Quant à l'utilisation de la variété, le meilleur taux de survie a été obtenu avec la variété locale. Toutefois l'interaction entre traitement et variété a eu des effets significatifs à toutes les dates d'observations sauf à 45 jours où l'effet était non significatif (Tableau 2).

Tableau 2 :Taux de reprise 7 jour et taux de survie à 15, 30, 45 jours (laitue)

\begin{tabular}{|l|l|c|l|l|l|}
\hline Variété & Traitement & TR7 & TS15 & TS30 & TS45 \\
\hline \multirow{4}{*}{ Améliorée } & T1 & $25 \pm 50$ & $25 \pm 50$ & $0 \pm 0$ & $0 \pm 0$ \\
\cline { 2 - 6 } & T2 & $25 \pm 50$ & $25 \pm 50$ & $25 \pm 50$ & $25 \pm 50$ \\
\cline { 2 - 6 } & T3 & $100 \pm 0$ & $100 \pm 0$ & $100 \pm 0$ & $75 \pm 50$ \\
\cline { 2 - 6 } & T4 & $100 \pm 0$ & $100 \pm 0$ & $100 \pm 0$ & $0 \pm 0$ \\
\hline \multirow{4}{*}{ Localenne } & T1 & $62.50 \pm 50.00$ & $62.50 \pm 50.0$ & $56.25 \pm 51.25$ & $25 \pm 44.72$ \\
\cline { 2 - 6 } & T2 & 0 & $0 \pm 0$ & $0 \pm 0.00$ & $0 \pm 0$ \\
\cline { 2 - 6 } & T3 & $100 \pm 0$ & $100 \pm 0$ & $100 \pm 0.00$ & $100 \pm 0$ \\
\cline { 2 - 6 } & T4 & $100 \pm 0$ & $100 \pm 0$ & $100 \pm 0.00$ & $75 \pm 50$ \\
\hline Moyenne & $68.75 \pm 47.87$ & $75 \pm 50$ & $50 \pm 57.74$ & $25 \pm 50$ \\
\hline Effet traitement & 0.0000 & 0.0000 & $0.02 .50 \pm 50.00$ & $50 \pm 51.64$ \\
\hline Effet Variété & 0.56908 & 0.56908 & 0.51892 & 0.00045 \\
\hline Interaction & 0.00948 & 0.00948 & 0.00122 & 0.05694 \\
\hline
\end{tabular}

Nombre des feuilles : Les résultats de l'analyse de la variance(ANOVA) révèlent qu'il existe de différences hautement significatives entre les traitements $(P=0,000)$, en ce qui concerne le nombre de feuille de la laitue à toutes les dates d'observations; Par rapport à l'utilisation de types de sols, le nombre des feuilles les plus élevé a été obtenu avec le sol T3, les sols de T2, T4 ont présentés le nombre des feuilles moyens comparativement au sol T1 qui avait présenté le nombre des feuilles nul. Quant à l'utilisation de la variété, l'analyse de la variance a montrer des différences significatives entre les traitements au 15 et 45 jours sauf à 30 jours où l'effet variété n'a pas révéler des différences significatives, le nombre de feuilles le plus élevé a été obtenu avec la variété locale par ailleurs l'interaction entre le traitement et la variété est significative pour le paramètre nombre des feuilles à 15 et 30 jours $(p=0,009)$; alors que elle est non significative pour le nombre de feuilles à 45 jours $(p=0,06)$ ( Tableau 3 ).

Tableau 3: Nombre des feuilles à 15, 30, 45 jours

\begin{tabular}{|l|l|l|l|l|}
\hline Variété & Traitements & NBF15 & NBF30 & NBF45 \\
\hline Améliorée & T1 & $0.5 \pm 1.00$ & $0.00 \pm 0.00$ & $0.00 \pm 0.00$ \\
\cline { 2 - 5 } & T2 & $0.5 \pm 1.00$ & $1.00 \pm 2.00$ & $1.25 \pm 2.5$ \\
\cline { 2 - 5 } & T3 & $2.0 \pm 0.00$ & $4.75 \pm 1.89$ & $5.50 \pm 3.7$ \\
\cline { 2 - 5 } & T4 & $2.0 \pm 0.82$ & $3.75 \pm 1.26$ & $0.00 \pm 0.0$ \\
\hline Moyenne & $1.25 \pm 1.06$ & $2.38 \pm 2.42$ & $1.69 \pm 3.07$ \\
\hline
\end{tabular}




\begin{tabular}{|c|c|c|c|c|}
\hline $\begin{array}{l}\text { Mununga et al., } \\
\text { sols contaminés }\end{array}$ & $\begin{array}{l}\text { iosc } \\
\text { shhi ( }\end{array}$ & ur de croiss & deux variét & gumes ins \\
\hline Locale & $\mathrm{T} 1$ & $0.00 \pm 0.00$ & $0.00 \pm 0.00$ & $0.00 \pm 0.00$ \\
\hline & $\mathrm{T} 2$ & $4.00 \pm 0.00$ & $6.00 \pm 1.63$ & $7.25 \pm 1.71$ \\
\hline & T3 & $4.25 \pm 1.89$ & $6.00 \pm 2.00$ & $6.00 \pm 4.32$ \\
\hline & T4 & $2.00 \pm 1.63$ & $1.25 \pm 1.50$ & $1.00 \pm 2.00$ \\
\hline Moyenne & & $2.56 \pm 2.10$ & $3.31 \pm 3.11$ & $3.56 \pm 3.93$ \\
\hline Effet traitement & & 0.00014 & 0.0000 & 0.0000 \\
\hline \begin{tabular}{|l|l|l|} 
Effet Variété \\
\end{tabular} & & 0.00177 & 0.090513 & 0.0358 \\
\hline Interaction & & 0.00246 & 0.000458 & 0.0670 \\
\hline
\end{tabular}

Hauteur des plantes: Les résultats de l'analyse de la variance révèlent des différences significatives entre les traitements $(p=0,000)$ pour ce qui est de la hauteur des plantes à toutes les dates d'observations. Par rapport à l'utilisation des types de sols, la meilleure croissance a été obtenue avec le sol de Kipushi, les sols de Kassapa et Ruashi ont présenté une croissance moyenne, alors que la petite croissance des plantes a été obtenue avec les sols de Pengapenga. L'analyse de la variance montre que l'effet variété a influencé significativement la croissance à toutes les dates d'observation, sauf pour la croissance à 30 jours et cela pour toutes les deux variétés. Quant à l'utilisation de la variété, la meilleure croissance a été obtenue avec la variété locale. C'est ainsi que l'interaction entre traitement et variété a eu des effets significatifs à toutes les dates d'observations sauf à 45 jours où l'effet a été non significatif(Tableau4).

Tableau 4: Hauteur des plantes à $15,30,45$ jours

\begin{tabular}{|l|l|c|c|c|}
\hline Variété & Traitement & HPL15 & HPL30 & HPL45 \\
\hline \multirow{5}{*}{ Améliorée } & T1 & $0.25 \pm 0.50$ & $0.00 \pm 0.00$ & $0.00 \pm 0.00$ \\
\cline { 2 - 5 } & T2 & $0.25 \pm 0.50$ & $0.50 \pm 1.00$ & $1.00 \pm 2.00$ \\
\cline { 2 - 5 } & T3 & $1.50 \pm 1.00$ & $5.75 \pm 2.50$ & $6.25 \pm 4.19$ \\
\cline { 2 - 5 } & T4 & $1.50 \pm 0.58$ & $3.00 \pm 0.82$ & $0.00 \pm 0.00$ \\
\hline Moyenne & T1 & $0.88 \pm 0.89$ & $2.31 \pm 2.68$ & $1.81 \pm 3.39$ \\
\hline Locale & T2 & $0.00 \pm 0.00$ & $0.00 \pm 0.00$ & $0.00 \pm 0.00$ \\
\cline { 2 - 5 } & T3 & $2.25 \pm 0.50$ & $6.75 \pm 3.20$ & $8.75 \pm 4.35$ \\
\cline { 2 - 5 } & T4 & $1.50 \pm 1.00$ & $6.50 \pm 2.38$ & $7.25 \pm 5.44$ \\
\hline Moyenne & $1.00 \pm 0.82$ & $1.25 \pm 1.50$ & $1.00 \pm 2.00$ \\
\hline Effet traitement & 0.0000 & $3.62 \pm 3.67$ & $4.25 \pm 5.09$ \\
\hline Effet Variété & 0.002623 & 0.0000 & 0.000263 \\
\hline Interaction & 0.000688 & 0.05082 & 0.032783 \\
\hline
\end{tabular}

Germination et Survie d'Albemoschus esculentus : Les résultats de l'analyse de la variance montrent qu'il existe des différences significatives entre les traitements $(p=0,000)$ pour ce qui est de germination à 8 jours ainsi que de la survie à toutes les dates d'observations. Par rapport à l'utilisation des types de sols, le meilleur taux de germination (100\%) a été obtenu avec le sol de Kassapa, les sols Kipushi et Ruashi ont présenté des taux de germination intermédiaires, alors que le taux de germination le plus faible a été obtenue avec les sols de Pengapenga. L'analyse de la variance montre que l'effet variété n'a pas influencé significativement la levée à toutes les dates d'observation et cela pour toutes les deux variétés. Quant à la variété le meilleur taux de germination a été obtenu avec la variété locale. Cependant l'interaction entre traitement et variété n'a pas influencé significativement la germination à 8 jours $(p>0.05)$. Par rapport à l'utilisation des types de sols, le meilleur taux de survie a été obtenu avec le sol de Kassapa, les sols de Kipushi et Ruashi ont présenté des taux de survie intermédiaires, alors que le taux de survie le plus faible a été obtenue avec le sol de Pengapenga. L'effet variété n'a pas influencé significativement la survie du gombo à toutes les dates des semis. Quant à l'utilisation de la variété, le meilleur taux de survie a été obtenu avec la variété V1. Toutefois l'interaction entre traitement et variété a eu des effets significatifs à toutes 


\section{Mununga et al., J. Appl. Biosci. 2018 Vigueur de croissance de deux variétés de légumes installées sur sols contaminés à Lubumbashi (RD Congo).}

les dates d'observations sauf à 45 jours où l'effet était non significatif (Tableau 6).

Tableau 6 : Taux de germination et survie de plantules d'Albemoschus esculentus T1 : sol de Kassapa, T2 : sol de Pengapenga, T3 : sol de Kipushi, T4 : sol de Ruashi.

\begin{tabular}{|l|l|l|l|l|}
\hline variété & Traitement & TG8 & TS30 & TS60 \\
\hline \multirow{4}{*}{ V1 } & T1 & $100 \pm 0.00$ & $100 \pm 0.00$ & $100 \pm 0.00$ \\
\cline { 2 - 5 } & T2 & $0.00 \pm 0.00$ & $0.00 \pm 0.00$ & $0.00 \pm 0.00$ \\
\cline { 2 - 6 } & T3 & $50 \pm 0.96$ & $50 \pm 0$ & $50 \pm 0$ \\
\cline { 2 - 6 } & T4 & $75 \pm 0.58$ & $75 \pm 0$ & $75 \pm 0$ \\
\hline \multirow{4}{*}{ Voyenne } & T1 & $25.81 \pm 1.28$ & $25.50 \pm 50.00$ & $25.50 \pm 50.00$ \\
\cline { 2 - 6 } & T2 & $100 \pm 1.26$ & $100 \pm 0$ & $100 \pm 0$ \\
\cline { 2 - 6 } & T3 & $0.00 \pm 0.00$ & $0.00 \pm 0.00$ & $0.00 \pm 0.00$ \\
\cline { 2 - 6 } & T4 & $75 \pm 1.41$ & $75 \pm 0$ & $75 \pm 0$ \\
\hline Moyenne & & $25.00 \pm 0.82$ & $25 \pm 50$ & $25 \pm 50$ \\
\hline Effet traitement & & $0.56 \pm 1.71$ & $50 \pm 0.87$ & $50 \pm 0.87$ \\
\hline Effet variété & & 0.400 & 0.0000 & 0.0000 \\
\hline Interaction & & 0.132 & 0.56908 & 0.56908 \\
\hline
\end{tabular}

Nombre des feuilles : Les résultats de l'analyse de la variance(ANOVA) révèlent qu'il existe de différences hautement significatives entre les traitements $(P=0,000)$, en ce qui concerne le nombre de feuille du gombo à toutes les dates d'observations. Par rapport à l'utilisation des types de sols, le nombre de feuille le plus élevé a été obtenu avec le sol de Kassapa, les sols Kipushi et Ruashi ont présenté le nombre de feuilles intermédiaires, alors que le nombre de feuille était nul avec les sols de Pengapenga. Quant à l'utilisation de la variété l'analyse de la variance montre que l'effet variété a influencé significativement le nombre de feuille à 30 jours sauf à 60 jours où l'effet variété était non significatif. Les sols de Kassapa et Ruashi ont donné le nombre des feuilles le plus élevé, le sol de Kipushi avait présenté le nombre des feuilles moyen, alors que le sol de T2 avait donné un nombre des feuilles nul. Par ailleurs l'interaction entre le traitement et la variété est non significative pour le nombre des feuilles ( $p>0.05$ ) (Tableau 7).

Tableau7 : Nombre des feuilles de plantules d'Albemoschus esculentus T1 : sol de Kassapa, T2 : sol de Pengapenga, T3 : sol de Kipushi, T4 : sol de Ruashi.

\begin{tabular}{|l|l|l|l|}
\hline variété & Traitement & NBF 30 & NBF 60 \\
\hline \multirow{4}{*}{ V1 } & T1 & $3.25 \pm 0.50$ & $6.0 \pm 2.31$ \\
\cline { 2 - 4 } & T2 & $0.00 \pm 1.00$ & $0.0 \pm 0.00$ \\
\cline { 2 - 4 } & T3 & $3.50 \pm 129$ & $4.5 \pm 1.00$ \\
\cline { 2 - 4 } & T4 & $4.50 \pm 0.58$ & $8.0 \pm 1.83$ \\
\hline Moyenne & $2.94 \pm 1.73$ & $4.62 \pm 3.34$ \\
\hline \multirow{4}{*}{ 2 } & T1 & $3.75 \pm 0.50$ & $5.00 \pm 0.82$ \\
\cline { 2 - 4 } & T2 & $0.00 \pm 0.00$ & $0.00 \pm 0.00$ \\
\cline { 2 - 4 } & T3 & $2.00 \pm 1.41$ & $4.50 \pm 3.11$ \\
\cline { 2 - 4 } & T4 & $2.50 \pm 1.91$ & $4.70 \pm 3.77$ \\
\hline Moyenne & & $2.06 \pm 1.77$ & $3.56 \pm 3.08$ \\
\hline Effet traitement & & 0.000 & 0.00 \\
\hline Effet variété & & 0.029 & 0.369 \\
\hline Interaction & 0.120 & 4.28 \\
\hline
\end{tabular}

Croissance et poids de fruits: Les résultats de l'analyse de la variance révèlent des différences significatives entre les traitements $(p=0,000)$ pour ce qui est de la hauteur à 30,60 jours et du poids à 60 des 
plantes du gombo. Par rapport à l'utilisation des types de sols, la meilleure croissance a été obtenue avec le sol de Kassapa et Ruashi, les sols de Kipushi avait présenté une croissance moyenne, alors que la petite croissance des plantes a été obtenue avec les sols de Pengapenga. Quant à l'utilisation de la variété, l'analyse de la variance montre que l'effet variété a influencé significativement la croissance à toutes les dates d'observation. La meilleure croissance a été obtenue avec la variété locale. Cependant l'interaction entre traitement et variété n'a pas influencé significativement la croissance des plantes. L'effet variété a influencé significativement la croissance du gombo à toutes les dates d'observation. Par rapport à l'utilisation des types de sols, le plus grand poids a été obtenu avec le sol de Kassapa, Ruashi, les sols de Kipushi avait présenté un poids moyen, alors que le faible poids a été obtenu avec les sols de T2 Pengapenga. Quant à l'utilisation de la variété, l'analyse de la variance montre que l'effet variété n'a pas influencé significativement le poids des fruits à 60 jours. La meilleure croissance a été obtenue avec la variété locale. Cependant l'interaction entre traitement et variété n'a pas influencé significativement la le poids de fruits du gombo. (Tableau 8).

Tableau 8: Hauteur et poids de fruits de plantules d'Albemoschus esculentus T1: sol de Kassapa, T2: sol de Pengapenga, T3 : sol de Kipushi, T4 : sol de Ruashi.

\begin{tabular}{|l|l|l|l|l|}
\hline variété & Traitement & HPL 30 & HPL 60 & PDF60 \\
\hline \multirow{4}{*}{ V1 } & T1 & $17.32 \pm 0.39$ & $26.00 \pm 2.31$ & $2.38 \pm 2.77$ \\
\cline { 2 - 5 } & T2 & $2.00 \pm 4.00$ & $0.00 \pm 0.00$ & $0.00 \pm 0.00$ \\
\cline { 2 - 5 } & T3 & $11.53 \pm 4.76$ & $18.50 \pm 5.57$ & $1.75 \pm 2.06$ \\
\cline { 2 - 5 } & T4 & $16.05 \pm 6.05$ & $24.35 \pm 6.65$ & $2.51 \pm 2.09$ \\
\hline Moyenne & T1 & $11.72 \pm 6.81$ & $17.21 \pm 11.39$ & $1.66 \pm 2.08$ \\
\hline \multirow{4}{*}{ V2 } & T2 & $0.00 \pm 0.00$ & $0.00 \pm 0.00$ & $1.52 \pm 3.05$ \\
\cline { 2 - 5 } & $\mathrm{T} 3$ & $10.18 \pm 0.19$ & $10.25 \pm 6.85$ & $3.00 \pm 0.00$ \\
\cline { 2 - 5 } & $\mathrm{T} 4$ & $7.60 \pm 6.05$ & $15.50 \pm 4.65$ & $6.36 \pm 4.24$ \\
\hline Moyenne & $7.14 \pm 5.21$ & $9.56 \pm 8.01$ & $2.75 \pm 3.56$ \\
\hline Effet traitement & 0.000 & 0.00 & 0.015 \\
\hline Effet variété & 0.000 & 0.00 & 0.230 \\
\hline Interaction & 0.083 & 0.103 & 0.281 \\
\hline
\end{tabular}

\section{DISCUSSION}

Concentration en ETMs dans les différents sols de jardins potagers de la région de Lubumbashi : Des résultats d'analyse de laboratoire, il ressort que plus de $75 \%$ des sols échantillonnés sont contaminés en cuivre, le sol de la Rwashi a présenté des teneurs élevées 20 fois supérieure que la norme, alors que le sol de Pengapenga dépasser la valeur seuil 3 fois, ce phénomène s'expliquerais par les fait que ces différents sols sont proches des activités minières (Macnair, 2003). Des situations similaires ont été observées par (Macnair, 2003) qui avait montré que la contamination de sol était fonction des activités minières pratiquées dans cette région de. Contrairement au sol de Kassapa qui avait présenté des teneurs excédant légèrement la valeur normale, mais le sol de Kipushi considérer comme contaminé au départ n'avait fait preuve d'aucune trace de cuivre dans ce sol. Par ailleurs, la contamination a été démontré qu'elle est réelle dans la ville de Lubumbashi, dans le rapport du Centre Carter (2012), qui a montré qu'une pollution de l'eau, du sol et des plantes comestibles était liée à l'exploitation minière du cuivre et du cobalt par les entreprises minières Chemaf et Ruashi Mining installées respectivement à Tshamilemba et Kabetsha dans la ville de Lubumbashi. Ce centre Carter montrent qu'après analyses des échantillons dans un laboratoire, l'eau de puits, le sol ainsi que les plantes comestibles prélevés dans ces deux quartiers, ont présenter des fortes concentrations en plomb, cadmium et zinc largement supérieures à la normale, des mêmes études effectuées par (Mpundu, 2010) dans la région de Lubumbashi, ont montrées que les sols, les légumes vendus dans différents marchés de ladite ville contenaient des teneurs excédant la norme établie par Coppenet, et al., 1993). Les études effectuées par Winterhalder (1996) cité par Linxian et al., (2013), dans la région de Sudbury avaient montrées que les activités minières (fonderie de Cu-Ni) avait conduit à la disparition de plus de 36000 hectares de forets naturelles et à la dénudation de plus 
de 10000 hectares. Toutefois des situations similaires ont été observées par les études effectuées par Kikilä (2003) dans la région de Harjavalta (En Finlande) qui avait montré que les activités minières notamment la fonderie de cuivre et nickel était responsables de la dégradation de la diversité des espèces et du couvert végétal de pin sylvestre. La contamination de la chaine alimentaire est un problème réel dans la province du Haut-Katanga, les études réalisée par (Vande Weghe et al., 2005), nous renseignent que le bassin de la Lufira contient des teneurs très élevées en etms notamment (Cuivre, Zinc, Arsenic, Plomb, Cadmium et Cobalt). Le centre caryer confirme que la contamination dans la région de Lubumbashi est réelle, partant de ces résultats, il ressort que l'eau, le sol ainsi que les plantes liée à l'exploitation du cuivre et du cobalt par les entreprises Chemaf et Ruashi Mining à Tshiamilemba et Kabetsha présentés des teneurs largement supérieur au seuil de toxicité.

Comportement des plantules de deux variétés d'Abelmoschus esculentus et de Lactuca sativa installées sur substrats riche en cuivre : Des résultats de l'analyse de la variance, il ressort les types de sol pour les paramètres végétatifs tels que la reprise, la survie, ainsi que le nombre de feuilles de la laitue ont montrer des différences significatives entre les traitements, nos résultats infirment ceux obtenus par Duprat-Invernizzi et al., 2006 ; Mbonigaba, 2007 qui ont montrer que l'apport de la bouse des vaches aux sol contaminés de Tshamilemba n'a pas influencer significativement la germination de la laitue. En effet, la croissance des plantes sur des sols ayant des concentrations très élevées en ETMs est limitée et dans la plupart des cas, la morphologie de ces plantes est altérée car les ETMs affectent le métabolisme des plantes et dans certains cas, inactiver les constituants de membranes dans les cellules. Cependant, les concentrations élevées des ETMs, peuvent causer des effets visibles de phytotoxicité (Kabata-Pendias A, 2001). La laitue a présenté un très faible taux de survie sur les sols contaminés de Pengapenga (T1) et ceux de Luwowoshi (T4) d'une manière générale pour toutes les deux variétés (locale et améliorée) à 45 jours par rapport aux sols des Kassapa (T2) et ceux de Kipushi (T3) pour les deux variétés hormis $T 4$ à 15 et 30 jours où il a été bon pour toutes les deux variétés dont la variété locale était la meilleure. En effet, les deux variétés ont montrés une sensibilité pour les fortes teneurs en ETMs des sols contaminés de Pengapenga et Rwashi (Luwowoshi) comparativement aux sols Kipushi et celui de Kassapa. Cependant une mortalité de $100 \%$ a été observée sur le sol Pengapenga à 45 jours pour toutes les variétés. Le nombre des feuilles de la laitue a été meilleur pour les sols Kassapa et Kipushi pour toutes les deux variétés dont le grand nombre des feuilles était de la variété locale. Et pour Pengapenga et Luwowoshi qui ont produit des plantes chétives à petites tailles dont certaines plantes n'ont pas survécu à 45 jours et par conséquent il n'y avait plus des feuilles car les plantes étaient mortes déjà à 45 jours mais la variété améliorée a donné des feuilles sur le sol Luwowoshi à 15 et 30 jours inferieur à 4 . Ceci s'expliquerait probablement par le fait que pour T4, malgré des fortes concentrations en ETMs, le niveau haut $\mathrm{du} \mathrm{pH}$ a induit une faible mobilité des éléments nutritifs dans le sol. Généralement après analyse de la variance, les résultats montrent que qu'il y avait des différences significatives entre les traitements pour ce qui est de la levée à 8 jours ainsi que pour la survie des plantes du gombo, ceci s'expliquerait par les faits que la germination des graines est liée non seulement à des facteurs intrinsèques de la graine mais aussi des facteurs extrinsèques tels que l'eau et la température qui sont très capitale pour amorcer le processus de germination (Useni et al., 2013). Nos résultats réfutent ceux trouvés par Mpundu et al., 2014 ; Mpundu et al., 2017 pour ce qui est de la survie du gombo; ceci serait dues au fait que la plante du gombo tolère la contamination cuprique même à des fortes concentrations de cuivre (Mpundu et al., 2013). Contrairement aux études effectuées par Kloke, 1979 qui ont montrés que les sols contaminés en éléments traces métalliques peuvent produire des plantes d'apparence normale alors que la concentration de ces même ETM dans le tissus peut avoir l'impact sur la croissance, pour la consommation animal. Au 60ème jour le meilleur résultat a été observé avec la variété Afrisem. Cette situation peut toutefois s'expliquer probablement par le fait que pour ces traitements NPK apporté à favoriser l'augmentation du $\mathrm{pH}$ qui, généralement immobilise les éléments trace métalliques et cette augmentation favorise par ailleurs une forte mobilité des éléments nutritifs (juste et al., 1995). Par rapport au nombre de feuilles, aucune différence significative n'a pas été signalé lors de nos premières observations à part le sol de Pengapenga qui n'a pas répondu dû à une forte concentration de cuivre. Ceci serait dû au fait que dans l'emploi de l'amendement, il y a eu une limite d'utilisation de cette dernière. En effet, au-delà d'un certain seuil, le développement des végétaux devient difficile suite au phénomène de complication et blocage (Mobambo, 2007 ; 2008). Pour le paramètre poids des fruits, le résultat obtenu était similaire à ceux obtenus par d'autres chercheurs, tels que Kabila, 2013, l'analyse de la variance avait montré des différences significatives ce 
phénomènes pourrait être expliqué par le fait que la présence excessive d'éléments contaminants pousse la plante à utiliser la grande quantité de son énergie à la tolérance de stress. Ces résultats vont des paires avec ceux obtenus par Temgoua .E, et al., 2015, qui ont trouvés que la contamination de sol n'avait pas influencé significativement la survie des plantules des différents légumes dans la région de Dschang (Cameroun).

Risques de contamination de la chaine alimentaire des plantules de d'Abelmoschus esculentus et de Lactuca sativa: Des tous les sols utilisés pour notre expérimentation, le sol de Ruashi présente de teneurs élevées en cuivre, suivi de celui de Pengapenga, puis du sol de Kassapa et le sol de Kipushi n'avait aucune trace de cuivre dans le sol ; c'est ainsi seules les plantes ayant survécu sur le sol de Kassapa et Kipushi ont fait objet des analyses chimiques. De ces résultats, il ressort que la laitue a beaucoup accumulé le cuivre contenue dans le sol de Kassapa, ce phénomène s'explique par le fait que la laitue est douée de cette capacité d'accumuler beaucoup les éléments traces. Nos résultats se rapportent avec ceux trouvés par Temgoua .E, et al., 2015 qui ont trouvés des teneurs 2 fois supérieures à la norme pour les laitues cultivées sur substrat contaminé dans la région de Dschang (Cameroun). Des études

\section{CONCLUSION}

Les résultats ont montrés que la contamination de la chaine alimentaire dans la ville de Lubumbashi est réelle, des fortes concentrations retrouvées dans les parties aériennes de la laitue, montre le vrai danger que présente les différents sols dans la région de Lubumbashi à transférer les ETMs dans les plantes et à affecter les consommateurs des plantes cultivées sur sols

\section{RÉFÉRENCES BIBLIOGRAPHIQUES}

Alegria, A., Barbera, R., Errecalde, F., Farre, R. and Largada, M.J. 1992. Relationship between cobalt, copper and zinc content of soil and vegetable. Nahrung 36 (5): 451-460.

Malaisse François, Alan JM. Baker, Sophie Ruelle., 1999. Diversity of plant communities and leaf heavy metal content at Luiswishi copper/cobalt mineralization, Upper Katanga, Dem. Rep. Congo Biotechnol. Agron. Soc. Environ. 19993 (2), 104-114. similaires effectuées par (Ntangmo Tsafack et al., 2012) ont montrées que l'arrosage de la culture de laitue avec de l'eau contenant moins des etms ont trouvés de fortes teneurs de cuivre dans les feuilles de la laitue, cette contamination a été attribuée à la contamination du sol. Cependant, les légumes cultivés sur des sols contaminés en ETMs peuvent accumuler des teneurs élevées qui peuvent constituer des risques de santé énormes chez les consommateurs (Islam et al., 2007 ; Bouchouata et al., 2011). Les plantes prélèvent des métaux lourds dans les sols ou par exposition aux poussières dans les différentes parties de la plante à l'air en provenance des sites pollués (Zurera-Cosano et al., 1989). Les études effectuées par Alegria et al., 1992 ont montrées les relations entre la concentration du cobalt, du cuivre, et du zinc dans sol et celles des légumes.de ces résultats, ils ont obtenu des corrélations significatives entre les concentrations totales et extractibles du cobalt du sol et celle des racines d'une part, et entre les concentrations totales du cuivre dans le sol et celles retrouvées dans les parties aériennes. Des études similaires effectuées au Kenya par Mutune, A. N. et al., 2014, ont démontres que les différents légumes produits sur le sol contaminé en zinc, cuivre, cobalt ont engendré à la contamination des légumes.

contaminés en cuivre. C'est ainsi nous suggérerons qu'une application des amendements organiques et calcaires aux sols riches en cuivre soient encouragée par les décideurs politiques en vue de diminuer la mobilité des ETMs dans le sol et de réduire le transfert des ETMs vers les plantes.

Baize et Tercet, 2002. Les éléments traces métalliques dans les sols. Approches fonctionnelles et spatiales. INRA Editions, Paris, $565 \mathrm{p}$.

Bouchouata, O., Jaafar, B., Bounakhla, M.,Doukkali, A. and Attarassi, B. 2011. Étude de la contamination par les métaux lourds des eaux d'irrigation et les cultures maraîchères dans la zone M'nasra (Gharb, Maroc), Science Lib Editions Mersenne, 3, No 111006 ISSN 21114706.

Bourrelier P.H., Berthelin J., 1998. Contamination des sols par les éléments traces : les risques et leur 
gestion. Rapport $n^{\circ} 42$ Académie des sciences. $439 \mathrm{p}$.

Bruneau, 1983. Effet d'amendements carbonatés et organiques sur la culture de deux légumes sur sol contaminé à Lubumbashi (RD Congo).

Centre Carter 2012. Les Investissements Miniers en République Démocratique du Congo : Développement ou Appauvrissement des Communautés Locales? Rapport d'impact des investissements miniers étrangers sur les droits humains Cas des investissements Chemical of Africa (Chemaf) et Ruashi Mining au Katanga Octobre 2012

Cheverry C., 1994. La dégradation chimique des sols en Bretagne. Étude et Gestion des sols, $1: 7-21$

Coppenet M., Golven J., Simon J.-C., Le Corre L., Le Roy M., 1993. Évolution chimique des sols en exploitations d'élevage intensif : exemple du Finistère. Agronomie, 13: 77- $83 p$.

Islamul, E., Yang, X., He, Z. and Mahmood, Q. 2007. Assessing potential dietary toxicity of heavy metal in selected vegetables and food crops. $J$. Zhejiang Univ. Sci. B. 8 (1): 1-13.

Juste C, Chassin P, Gomez A, Linères M, Mocquot B., Feix I., et Wiart J., 1995. Les micropolluants métalliques dans les boues résiduaires des stations d'épuration urbaines. In Collection «Valorisation agricole des boues d'épuration », Connaitre pour agir. Adème, ISBM 2-86817-100-1,209 p.

Kabata-Pendias A. \& Pendias H., 2001. Trace elements in soils and plants. 3rd ed. Boca Raton, FL, USA : CRC Press.

Kiikkilä O. 2003. Heavy-metal pollution and remediation of forest soil around the Harjavalta $\mathrm{Cu}-\mathrm{Ni}$ smelter, in SW Finland. Silva Fennica. 37(3), 399- 415.

Kloke, A., 1979. Content of arsenic, cadmium, chromium, fluorine, lead, mercury and nickel in plants grown on contaminated soil. Paper presented at United Nations - ECE Symp

Leteinturier B. \& Malaisse F., 1999. De la réhabilitation des sols pollués par l'activité minière du cuivre en Afrique centro-australe. Bull. Séance Acad. R. Sci. Outre-Mer, 45(4), 535-554.

Mench, M. et Baize, D. 2004. Contamination des sols et de nos aliments d'origine végétale par les éléments en traces mesures pour réduire l'exposition, Courrier de l'environnement de I'INRA N52. 26p.
Macnair MR. 2003. The Hyper accumulation of Metals by Plants. Advances in Botanical Research 40:65-105p

Mbonigaba M.J.J., 2007. Étude de l'impact des composts à base de biomasse végétale sur la dynamique des indicateurs physico-chimiques, chimiques et microbiologiques de la fertilité des sols : application sur trios sols acides tropicaux du Rwanda. Thèse de doctorat, FUSAGx, Gembloux, 243p.

Mougeot et Moustier., 2004. Les dynamiques de l'agriculture urbaine: caractérisation et évaluation In: Smith.O, Moustier, P., Mougeot, L. et Fall, A. Développement durable de l'agriculture urbaine en Afrique francophone. Enjeux, concepts et méthodes, CIRAD et CRDI. pp. 23-29.

Mpundu M.M., 2010. Contaminations des sols en Éléments Traces Métalliques à Lubumbashi (Katanga/RDCongo). Évaluation des risques de contamination de la chaîne alimentaire et choix de solutions de remédiation. Thèse de doctorat: Faculté des Sciences Agronomiques, Université de Lubumbashi (RD Congo).

Mpundu M. M., Useni S. Y., Mwamba M. T., Kateta M. G., Mwansa M., llunga K., Kamengwa K. C., K. K., Nyembo K. L., 2013b. Teneurs en éléments traces métalliques dans les sols de différents jardins potagers de la ville minière de Lubumbashi et risques de contamination des cultures potagères. Journal of Applied Biosciences 65:4957 - 4968

Mpundu M. M, Useni S. Y, Nyembo K. L, Colinet G., 2014. Effets d'amendements carbonatés et organiques sur la culture de deux légumes sur sol contaminé à Lubumbashi (RD Congo). Biotechnol. Agron. Soc. Environ. 18(2), 1-9.

Mpundu M.M, Mununga K.F, Kaumbu K. J-M, Mwilambwe K.X, Maloba K. J-P, Banza I.M, Mukunto K.I., 2017. Influence des sols contaminés en cuivre sur le développement de deux variétés (locale et améliorée) de légumes dans la région de Lubumbashi (RD. Congo). Journal of Applied Biosciences 115: 1141011422.

Ntangmo Tsafack et al., 2012. Physico-chemical and bacteriological quality of the vegetable watering water in the Dschang Town, Cameroon. Journal of Environnemental Protection 3: 949-955. 
Joe Nasr et Padilla Martine., 2004. Agricultures et urbanisation à l'est et au sud de la Méditerranée, Beyrouth : IFPO-DELTA, 2004, pp.391-405

Tembo DB, Sichilongo K, Cernak J 2006. Distribution of copper, lead, cadmium and zinc concentrations in soils around Kabwe Town in Zambia. Chemosphere, 63: 497-501.

Temgoua, E., Pfeifer, H.R. and Bitom, D. 2003. Trace element differentiation in ferruginous slope accumulation patterns of southern Cameroon, the role of climate change. Science of The Total Environment 303(3): 203 - 214.

Temgoua. E, H. Ntangmo Tsafack1, H.-R. Pfeifer2 and T. Njine., 2015. Teneurs en éléments majeurs et oligoéléments dans un sol et quelques cultures maraîchères de la ville de Dschang, Cameroun African Crop Science Journal, Vol. 23, No. 1, pp. $35-44$.
Useni Sikuzani Yannick, Chukiyabo Kibenge Minerve, Tshomba Kalumbu John, Muyambo Musaya Emmanuel, Kapalanga Kamina Prisca, Ntumba Ndaye François, Kasangij A Kasangij Patrick, Kyungu Kalilo, Baboy Longanza Louis, Nyembo Kimuni Luciens, Mpundu Mubemba Michel., 2013. Utilisation des déchets humains recyclés pour l'augmentation de la production du maïs (Zea mays L.) sur un ferralsol du sud-est de la RD Congo. Journal of Applied Biosciences 66:5070 - 5081 ISSN 1997-5902.

Zurera-Cosano, G., Moreno-Rojas, R., Salmeron-Egea, J. and Pozo Lora, R., 1989. Heavy metal uptake from greenhouse border soils for edible vegetable. Journal of the Science of Food and Agriculture 49(3): 307-314. 